\title{
Inverse Boundary Value Problem for a Fractional Differential Equations of Mixed Type with Integral Redefinition Conditions
}

\author{
T. K. Yuldashev ${ }^{* *}$ and B. J. Kadirkulov ${ }^{2 * *}$ \\ (Submitted by A. M. Elizarov) \\ ${ }^{1}$ Uzbek-Israel Joint Faculty of High Technology and Engineering Mathematics, \\ National University of Uzbekistan, Tashkent, 100174 Uzbekistan \\ ${ }^{2}$ Department of Mathematics and Information Technologies, \\ Tashkent State University of Oriental Studies, Tashkent, 100060 Uzbekistan \\ Received June 27, 2020; revised July 12, 2020; accepted August 3, 2020
}

\begin{abstract}
In this paper, we consider an inverse boundary value problem for a mixed type partial differential equation with Hilfer operator of fractional integro-differentiation in a positive rectangular domain and with spectral parameter in a negative rectangular domain. The differential equation depends from another positive parameter in mixed derivatives. With respect to first variable this equation is a fractional-order nonhomogeneous differential equation in the positive part of the considering segment, and with respect to second variable is a second-order differential equation with spectral parameter in the negative part of this segment. Using the Fourier series method, the solutions of direct and inverse boundary value problems are constructed in the form of a Fourier series. Theorems on the existence and uniqueness of the problem are proved for regular values of the spectral parameter. It is proved the stability of the solution with respect to redefinition functions, and with respect to parameter given in mixed derivatives. For irregular values of the spectral parameter, an infinite number of solutions in the form of a Fourier series are constructed.
\end{abstract}

DOI: $10.1134 / \mathrm{S} 1995080221030227$

Keywords and phrases: mixed type equation, inverse problem, parameters, solvability, Hilfer operator, Mittag-Leffler function, classical solution.

\section{STATEMENT OF THE INVERSE PROBLEM}

One of the most striking areas of mathematical analysis is the invention of fractional-order integrodifferential operators. Today, the theory and application of operators of fractional differentiation and integration have become a powerful industry of theoretical and applied research at the highest levels of different science and technology. At present, the operators of fractional differentiation and integration are also widely used in the study of problems associated with the study of the coronavirus COVID-19 (see, for example [1,2]).

In a rectangular domain $\Omega=\{(t, x):-a<t<b, 0<x<l\}$ we consider the fractional partial differential equation of mixed type

$$
0=\left\{\begin{array}{l}
\left(D^{\alpha, \gamma}-\nu \frac{\partial^{2}}{\partial x^{2}} D^{\alpha, \gamma}-\frac{\partial^{2}}{\partial x^{2}}\right) U(t, x)+f_{1}(t) g_{1}(x), \quad(t, x) \in \Omega_{1}, \\
\left(\frac{\partial^{2}}{\partial t^{2}}-\nu \frac{\partial^{4}}{\partial t^{2} \partial x^{2}}-\omega^{2} \frac{\partial^{2}}{\partial x^{2}}\right) U(t, x)+f_{2}(t) g_{2}(x), \quad(t, x) \in \Omega_{2},
\end{array}\right.
$$

where $\Omega_{1}=\{(t, x): 0<t<b, 0<x<l\}, \Omega_{2}=\{(t, x):-a<t<0,0<x<l\}, \nu$ is positive parameter, $\omega$ is positive spectral parameter, $a, b$ are positive real numbers,

$$
D^{\alpha, \gamma}=J_{0+}^{\gamma-\alpha} \frac{d}{d t} J_{0+}^{1-\gamma}, \quad 0<\alpha \leq \gamma \leq 1
$$

\footnotetext{
${ }^{*}$ E-mail: tursun.k.yuldashev@gmail .com

${ }^{* *}$ E-mail: kadirkulovbj@gmail.com
} 
is Hilfer operator and

$$
J_{0+}^{\alpha} \varphi(t)=\frac{1}{\Gamma(\alpha)} \int_{0}^{t} \frac{\varphi(\tau) d \tau}{(t-\tau)^{1-\alpha}}, \quad \alpha>0
$$

is Riemann-Liouville integral operator, $f_{1}(t) \in C[0 ; b], f_{2}(t) \in C[-a ; 0], g_{i}(x) \in C[0 ; l]$ are redefinition functions, $i=1,2$.

Problem $I \boldsymbol{T}_{\boldsymbol{\nu}, \boldsymbol{\omega}}$. It is required to find a triple of functions $\left\{U(t, x), g_{1}(x), g_{2}(x)\right\}$, which belongs to the class

$$
\left[\begin{array}{l}
t^{1-\gamma} \frac{\partial^{k} U}{\partial x^{k}} \in C\left(\bar{\Omega}_{1}\right), \quad \frac{\partial^{k} U}{\partial x^{k}} \in C\left(\bar{\Omega}_{2}\right), \quad D^{\alpha, \gamma} U \in C\left(\Omega_{1}\right) \\
U_{t t}, U_{x x} \in C\left(\Omega_{1} \cup \Omega_{2}\right), \quad k=0,1,2 \\
g_{i}(x) \in C[0 ; l], \quad i=1,2
\end{array}\right.
$$

satisfies equation ( 1 ) in the domain $\Omega_{1} \cup \Omega_{2}$, boundary value conditions

$$
\begin{aligned}
& \left.U(t, x)\right|_{x=0}=\left.U(t, x)\right|_{x=l}=0, \quad t \neq 0, \\
& U(-a, x)=U(b, x)+\varphi(x), \quad 0 \leq x \leq l,
\end{aligned}
$$

gluing conditions

$$
\lim _{t \rightarrow+0} J_{0+}^{1-\gamma} U(t, x)=\lim _{t \rightarrow-0} U(t, x), \quad \lim _{t \rightarrow+0} J_{0+}^{1-\alpha} \frac{d}{d t} J_{0+}^{1-\gamma} U(t, x)=\lim _{t \rightarrow-0} \frac{d}{d t} U(t, x)
$$

and additional integral conditions

$$
\begin{gathered}
\int_{0}^{b} \Theta_{1}(t) t^{1-\gamma} U(t, x) d t=\psi_{1}(x), \quad 0 \leq x \leq l, \\
\int_{-a}^{0} \Theta_{2}(t) U(t, x) d t=\psi_{2}(x), \quad 0 \leq x \leq l,
\end{gathered}
$$

where $\varphi(x), \psi_{i}(x)$ are given sufficiently smooth functions, $\Theta_{1}(t) \in C[0 ; b], \Theta_{2}(t) \in C[-a ; 0], i=1,2$.

The generalized integro-differentiation operator $D^{\alpha, \gamma}$ is a continuous interpolation of the well-known fractional order differentiation operators of Riemann-Liouville and Caputo, which describe diffusion processes [2, vol. 1, 47-85]. A physical and engineering interpretation of the generalized fractional operator $D^{\alpha, \gamma}$ is given in [2, vol. 4-8], [3-7]. In [4], in particular, were provide results on the existence and representation of solution to an initial value problem for the general ordinary linear fractional differential equation with generalized Riemann-Liouville fractional derivatives and constant coefficients by using operational calculus of Mikusinski type. In [8], the problem of source identification was studied for the generalized diffusion equation with operator $D^{\alpha, \gamma}$. In the work [9] the inverse problems are investigated for a generalized fourth-order parabolic equation with the operator $D^{\alpha, \gamma}$.

Note that boundary value conditions of the type (3) take place in modeling problems of the flow around a profile by a subsonic velocity stream with a supersonic zone. Nonlocal boundary value problems for different type of equations were studied in the works of many authors, in particular, in [10-16].

In our work, unlike mixed parabolic-hyperbolic equations, the problem of small denominators does not arise. In addition, in our solvability problem we impose conditions that are weaker than in the case of corresponding parabolic-hyperbolic equations. In this paper, we consider an inverse boundary value problem for a mixed type fourth-order differential equation with Hilfer operator of fractional integrodifferentiation and spectral parameter. The Fourier method of separation of variables is used taking into account the features of the fractional integro-differentiation operator. We study the solvability of the problem (1)-(7) for various values of the spectral parameter. We prove the stability of the solution with respect to redefinition functions and with respect to parameter given in mixed derivatives. This work is a further development and generalization of the results of [17-19]. 
We consider the Cauchy problem for a nonhomogeneous differential equation of fractional order

$$
\left\{\begin{array}{l}
D^{\alpha, \gamma} u(t)=k u(t)+f(t), \quad t \in\left(0, t_{1}\right) \\
\lim _{t \rightarrow+0} J_{0+}^{1-\gamma} u(t)=u_{0}
\end{array}\right.
$$

where $f(t)$ is given continuous function, $u_{0}=$ const.

Lemma 1. Let be $f(t) \in C\left(0 ; t_{1}\right) \cap L_{1}\left(0 ; t_{1}\right)$. Then the solution of the problem $(8) u(t) \in$ $C\left(0 ; t_{1}\right) \cap L_{1}\left(0 ; t_{1}\right)$ is represented as follows

$$
u(t)=u_{0} t^{\gamma-1} E_{\alpha, \gamma}\left(k t^{\alpha}\right)+\int_{0}^{t}(t-\tau)^{\alpha-1} E_{\alpha, \alpha}\left(k(t-\tau)^{\alpha}\right) f(\tau) d \tau,
$$

where

$$
E_{\alpha, \gamma}(z)=\sum_{m=0}^{\infty} \frac{z^{m}}{\Gamma(\alpha m+\gamma)}, \quad z, \alpha, \gamma \in \mathbb{C}, \quad \operatorname{Re}(\alpha)>0
$$

is Mittag-Leffler function [2, vol. 1, 269-295].

In [9], the Laplace method was applied to prove this Lemma 1. In [4] a solution was found using operational calculus for a more general problem than (8) in a specially constructed functional space. We note that there is a more rational way to solve this problem (8), which allows us to obtain an explicit solution (9) (see [20]).

\section{FORMAL EXPANSION OF THE SOLUTION OF THE DIRECT PROBLEM INTO FOURIER SERIES}

The solution of the differential equation (1) in the domain $\Omega$ is sought in the form of a Fourier series

$$
U(t, x)=\sum_{n=1}^{\infty} u_{n}(t) \vartheta_{n}(x)
$$

where

$$
u_{n}(t)=\int_{0}^{l} U(t, x) \vartheta_{n}(x) d x, \quad \vartheta_{n}(x)=\sqrt{\frac{2}{l}} \sin \mu_{n} x, \quad \mu_{n}=\frac{n \pi}{l}, \quad n \in \mathbb{N} .
$$

We suppose also

$$
g_{i}(x)=\sum_{n=1}^{\infty} g_{i n} \vartheta_{n}(x), \quad i=1,2
$$

where $g_{i n}=\int_{0}^{l} g_{i}(x) \vartheta_{n}(x) d x$. Substituting series (10) and (12) into equation (1), we obtain a countable systems of differential equations

$$
\begin{gathered}
D^{\alpha, \gamma} u_{n}(t)+\lambda_{n}^{2}(\nu) u_{n}(t)=f_{1}(t) g_{1 n}, \quad t>0, \\
u_{n}^{\prime \prime}(t)+\lambda_{n}^{2}(\nu) \omega^{2} u_{n}(t)=f_{2}(t) g_{2 n}, \quad t<0,
\end{gathered}
$$

where $\lambda_{n}^{2}(\nu)=\frac{\mu_{n}^{2}}{1+\nu \mu_{n}^{2}}, \mu_{n}=\frac{n \pi}{l}, n \in \mathbb{N}$. Taking (11) into account from the conditions (5) we obtain

$$
\lim _{t \rightarrow+0} J_{0+}^{1-\gamma} u_{n}(t)=\sqrt{\frac{2}{l}} \int_{0}^{l} \lim _{t \rightarrow+0} J_{0+}^{1-\gamma} U(t, x) \sin \mu_{n} x d x
$$




$$
\begin{gathered}
=\sqrt{\frac{2}{l}} \int_{0}^{l} \lim _{t \rightarrow-0} U(t, x) \sin \mu_{n} x d x=\lim _{t \rightarrow-0} u_{n}(t), \\
\lim _{t \rightarrow+0} J_{0+}^{1-\alpha} \frac{d}{d t} J_{0+}^{1-\gamma} u_{n}(t)=\sqrt{\frac{2}{l}} \int_{0}^{l} \lim _{t \rightarrow+0} J_{0+}^{1-\alpha} \frac{d}{d t} J_{0+}^{1-\gamma} U(t, x) \sin \mu_{n} x d x \\
=\sqrt{\frac{2}{l}} \int_{0}^{l} \lim _{t \rightarrow-0} \frac{d}{d t} U(t, x) \sin \mu_{n} x d x=\lim _{t \rightarrow-0} \frac{d}{d t} u_{n}(t) .
\end{gathered}
$$

Analogously we find from (4) that

$$
u_{n}(-a)=u_{n}(b)+\varphi_{n}
$$

where

$$
\varphi_{n}=\sqrt{\frac{2}{l}} \int_{0}^{l} \varphi(x) \sin \mu_{n} x d x, \quad n=1,2, \ldots
$$

Applying Lemma 1 to (13) and variation method of arbitrary constants to (14), we obtain the general forms of solutions

$$
\begin{gathered}
u_{n}(t, \nu)=A_{1 n} t^{\gamma-1} E_{\alpha, \gamma}\left(-\lambda_{n}^{2}(\nu) t^{\alpha}\right)+g_{1 n} h_{1 n}(t, \nu), \quad t>0, \\
u_{n}(t, \nu)=A_{2 n} \sin \lambda_{n}(\nu) \omega t+A_{3 n} \cos \lambda_{n}(\nu) \omega t+g_{2 n} h_{2 n}(t, \nu), \quad t<0,
\end{gathered}
$$

where $A_{i n}$ are arbitrary constants, $i=\overline{1,3}, n=1,2, \ldots$,

$$
\begin{gathered}
h_{1 n}(t, \nu)=\int_{0}^{t}(t-s)^{\alpha-1} E_{\alpha, \alpha}\left(-\lambda_{n}^{2}(\nu)(t-s)^{\alpha}\right) f_{1}(s) d s, \\
h_{2 n}(t, \nu)=\frac{1}{\lambda_{n}(\nu) \omega} \int_{0}^{t} \sin \lambda_{n}(\nu) \omega(t-s) f_{2}(s) d s .
\end{gathered}
$$

Taking into account that $h_{1 n}(0)=h_{2 n}(0)=0$ and satisfying functions (18) and (19) to conditions (15) and (16), we obtain the following systems of algebraic equations

$$
A_{2 n}=-\frac{\lambda_{n}(\nu)}{\omega} A_{1 n}, \quad A_{3 n}=A_{1 n} .
$$

Applying the condition (17) and representation (20) to (18) and (19), we derive

$$
A_{1 n}=\frac{\varphi_{n}+g_{1 n} h_{1 n}(b)-g_{2 n} h_{2 n}(-a)}{\Delta_{n}(\omega)}
$$

if there holds the condition

$$
\Delta_{0 n}(\omega, \nu)=\lambda_{n}(\nu) \omega^{-1} \sin \lambda_{n}(\nu) \omega a+\cos \lambda_{n}(\nu) \omega a-b^{\gamma-1} E_{\alpha, \gamma}\left(-\lambda_{n}^{2}(\nu) b^{\alpha}\right) \neq 0 .
$$

Substituting (21) into (20), for (18) and (19) we obtain the representations

$$
\begin{array}{ll}
u_{n}(t, \nu, \omega)=\varphi_{n} \eta_{1 n}(t, \nu, \omega)+g_{1 n} \eta_{2 n}(t, \nu, \omega)+g_{2 n} \eta_{3 n}(t, \nu, \omega), & t>0, \\
u_{n}(t, \nu, \omega)=\varphi_{n} \xi_{1 n}(t, \nu, \omega)+g_{1 n} \xi_{2 n}(t, \nu, \omega)+g_{2 n} \xi_{3 n}(t, \nu, \omega), & t<0,
\end{array}
$$

where

$$
\eta_{1 n}(t, \nu, \omega)=\frac{t^{\gamma-1}}{\Delta_{0 n}(\omega, \nu)} E_{\alpha, \gamma}\left(-\lambda_{n}^{2}(\nu) t^{\alpha}\right)
$$




$$
\begin{gathered}
\eta_{2 n}(t, \nu, \omega)=h_{1 n}(t, \nu)+h_{1 n}(b, \nu) \eta_{1 n}(t, \nu, \omega), \\
\eta_{3 n}(t, \nu, \omega)=-h_{2 n}(-a, \nu) \eta_{1 n}(t, \nu, \omega), \\
\xi_{1 n}(t, \nu, \omega)=\frac{1}{\Delta_{0 n}(\omega, \nu)}\left(\sin \lambda_{n}(\nu) \omega t+\cos \lambda_{n}(\nu) \omega t\right), \\
\xi_{2 n}(t, \nu, \omega)=h_{1 n}(b, \nu) \xi_{1 n}(t, \nu, \omega), \quad \xi_{3 n}(t, \nu, \omega)=h_{2 n}(t, \nu)+h_{2 n}(-a, \nu) \xi_{1 n}(t, \nu, \omega) .
\end{gathered}
$$

Substituting representations (23) and (24) into the Fourier series (10), we obtain

$$
\begin{aligned}
& U(t, x, \nu, \omega)=\sum_{n=1}^{\infty} \vartheta_{n}(x)\left[\varphi_{n} \eta_{1 n}(t, \nu, \omega)+g_{1 n} \eta_{2 n}(t, \nu, \omega)+g_{2 n} \eta_{3 n}(t, \nu, \omega)\right], \quad(t, x) \in \Omega_{1}, \\
& U(t, x, \nu, \omega)=\sum_{n=1}^{\infty} \vartheta_{n}(x)\left[\varphi_{n} \xi_{1 n}(t, \nu, \omega)+g_{1 n} \xi_{2 n}(t, \nu, \omega)+g_{2 n} \xi_{3 n}(t, \nu, \omega)\right], \quad(t, x) \in \Omega_{2} .
\end{aligned}
$$

To establish the uniqueness of the solution $U(t, x, \nu, \omega)$ of the direct problem (1)-(5) we suppose that there are two solutions $U_{1}$ and $U_{2}$ of this direct problem. Then their difference $U=U_{1}-U_{2}$ is a solution of equation (1), satisfying conditions (2)-(5) with functions $\varphi(x) \equiv 0$. Then for $\varphi_{n}=0$ it follows from representations (25) and (26) in the domain $\Omega$ that

$$
\begin{gathered}
\int_{0}^{l} t^{1-\gamma} U(t, x, \nu, \omega) \vartheta_{n}(x) d x=0, \quad(t, x) \in \Omega_{1}, \\
\quad \int_{0}^{l} U(t, x, \nu, \omega) \vartheta_{n}(x) d x=0, \quad(t, x) \in \Omega_{2} .
\end{gathered}
$$

Hence, by virtue of the completeness of the system of eigenfunctions $\left\{\vartheta_{n}(x)\right\}$ in $L_{2}(0 ; l)$, we deduce that $U(t, x, \nu, \omega) \equiv 0$ for all $x \in[0 ; l]$ and $t \in[-a ; b]$.

Since $t^{1-\gamma} U(t, x, \nu, \omega) \in C\left(\bar{\Omega}_{1}\right), U(t, x, \nu, \omega) \in C\left(\bar{\Omega}_{2}\right)$, then $t^{1-\gamma} U(t, x, \nu, \omega) \equiv 0$ in the domain $\bar{\Omega}$. Therefore, the solution of direct problem is unique in the domain $\bar{\Omega}$. Thus, we have proved that the following theorem holds:

Theorem 1. Suppose that there exists a solution of the direct problem (1)-(5). Then this solution is unique, if condition (22) is fulfilled for all $n \in \mathbb{N}$.

Now we consider the case, when condition (22) is violated. Let $\Delta_{0 m}(\omega, \nu)=0$ be for all $\omega, \gamma \in(0 ; 1)$. Then the direct problem $(1)-(5)(\varphi(x) \equiv 0)$ for fixed values of the redefinition functions has nontrivial solutions

$$
V_{m}(t, x)=v_{m}(t) \vartheta_{m}(x), \quad(t, x) \in \Omega,
$$

where

$$
v_{m}(t)=\left\{\begin{array}{l}
t^{\gamma-1} E_{\alpha, \gamma}\left(-\lambda_{m}^{2}(\nu) t^{\alpha}\right)+g_{1 n} h_{1 n}(t), \quad t>0, \\
\sin \lambda_{m}(\nu) \omega t+\cos \lambda_{m}(\nu) \omega t+g_{2 n} h_{2 n}(t), \quad t<0 .
\end{array}\right.
$$

From the condition $\Delta_{0 n}(\omega, \nu)=0$ we come to the trigonometric equation

$$
\sqrt{1+\frac{\lambda_{n}^{2}(\nu)}{\omega^{2}}} \sin \left(\lambda_{n}(\nu) \omega a+\rho_{n}\right)-b^{\gamma-1} E_{\alpha, \gamma}\left(-\lambda_{n}^{2}(\nu) b^{\alpha}\right)=0,
$$

where $\rho_{n}=\arcsin \left(\frac{\omega}{\sqrt{\omega^{2}+\lambda_{n}^{2}(\nu)}}\right)$. Hence we obtain, that the quantity $\Delta_{0 n}(\omega)$ vanishes at

$$
\omega=\frac{1}{\lambda_{n}(\nu) a}\left[(-1)^{k} \arcsin \frac{\omega b^{\gamma-1} E_{\alpha, \gamma}\left(-\lambda_{n}^{2}(\nu) b^{\alpha}\right)}{\sqrt{\omega^{2}+\lambda_{n}^{2}(\nu)}}+\pi k-\rho_{n}\right], \quad k=1,2, \ldots
$$


The set of positive solutions $\Im$ of trigonometric equation (28) with respect to spectral parameter $\omega$ is called a set of irregular values of the spectral parameter $\omega$. The set of the remaining all values of the spectral parameter $\aleph=(0 ; \infty) \backslash \Im$ is called a set of regular values of the spectral parameter $\omega$. For all regular values of the spectral parameter $\omega$ the quantity $\Delta_{0 n}(\omega, \nu)$ is nonzero. So, for large $n$ the values of $\Delta_{0 n}(\omega, \nu)$ can not become quite small and there the problem of "small denominators" does not arise. Therefore, for regular values of the spectral parameter $\omega$ the quantity $\Delta_{0 n}(\omega, \nu)$ is separated from zero.

Indeed, from the relations $\lambda_{n}^{2}(\nu)=\frac{\mu_{n}^{2}}{1+\nu \mu_{n}^{2}}, \mu_{n}=\frac{n \pi}{l}$ we see that $\lambda_{n}^{2}(\nu) \rightarrow \frac{1}{\nu}$ as $n \rightarrow \infty$. So, for regular values of the spectral parameter $\omega$ we have

$$
\lim _{n \rightarrow \infty} \Delta_{0 n}(\omega, \nu)=\frac{1}{\omega \sqrt{\nu}} \sin \frac{\omega}{\sqrt{\nu}} a+\cos \frac{\omega}{\sqrt{\nu}} a-b^{\gamma-1} E_{\alpha, \gamma}\left(-\frac{b^{\alpha}}{\nu}\right) \neq 0 .
$$

Lemma 2. Suppose that $\gamma \in(0 ; 1], a, b$ are arbitrary positive real numbers. Then for regular values of the spectral parameter $\omega$ and for arbitrary $n$ there exists a positive constant $M_{0}$ such that there holds the following estimate

$$
\left|\Delta_{0 n}(\omega, \nu)\right| \geq M_{0}>0 .
$$

Proof. From (28) for all $n$ and $a, b>0$ we derive

$$
\left|\Delta_{0 n}(\omega, \nu)\right| \geq\left|\sqrt{1+\frac{\lambda_{n}^{2}(\nu)}{\omega^{2}}}-b^{\gamma-1} E_{\alpha, \gamma}\left(-\lambda_{n}^{2}(\nu) b^{\alpha}\right)\right| \geq\left|1-b^{\gamma-1} E_{\alpha, \gamma}\left(-\lambda_{n}^{2}(\nu) b^{\alpha}\right)\right| .
$$

We use the following properties of the Mittag-Leffler function [2, vol. 1, 269-295]:

1) For all $k>0, \alpha, \gamma \in(0 ; 1], \alpha \leq \gamma, t \geq 0$ the function $t^{\gamma-1} E_{\alpha, \gamma}\left(-k t^{\alpha}\right)$ is completely monotonous and there holds

$$
(-1)^{n}\left[t^{\gamma-1} E_{\alpha, \gamma}\left(-k t^{\alpha}\right)\right]^{(n)} \geq 0, \quad n=0,1,2, \ldots
$$

2) For all $\alpha \in(0 ; 2), \gamma \in \mathbb{R}$ and $\arg z=\pi$ there takes place the following estimate

$$
\left|E_{\alpha, \gamma}(z)\right| \leq \frac{M_{1}}{1+|z|}
$$

where $0<M_{1}=$ const does not depend from $z$.

Then, from (30) and (31) implies that there exists a number $M_{0}$ such that

$$
\left|1-b^{\gamma-1} E_{\alpha, \gamma}\left(-\lambda_{n}^{2}(\nu) b^{\alpha}\right)\right|=M_{0}>0 .
$$

Consequently, for regular values of the spectral parameter $\omega$ there takes place (29): $\left|\Delta_{n}(\omega)\right| \geq M_{0}>0$. Lemma 2 is proved.

\section{REDEFINITION FUNCTIONS}

We apply the conditions (6) and (7) to Fourier series (25) and (26), respectively:

$$
\begin{gathered}
\psi_{1}(x)=\sum_{n=1}^{\infty} \vartheta_{n}(x) \int_{0}^{b} \Theta_{1}(t) t^{1-\gamma} \\
\times\left[\varphi_{n} \eta_{1 n}(t, \nu, \omega)+g_{1 n} \eta_{2 n}(t, \nu, \omega)+g_{2 n} \eta_{3 n}(t, \nu, \omega)\right] d t, \\
\psi_{2}(x)=\sum_{n=1}^{\infty} \vartheta_{n}(x) \int_{-a}^{0} \Theta_{2}(t) \\
\times\left[\varphi_{n} \xi_{1 n}(t, \nu, \omega)+g_{1 n} \xi_{2 n}(t, \nu, \omega)+g_{2 n} \xi_{3 n}(t, \nu, \omega)\right] d t .
\end{gathered}
$$


We suppose here that

$$
\psi_{i}(x)=\sum_{n=1}^{\infty} \psi_{i n} \vartheta_{n}(x), \quad i=1,2
$$

where $\psi_{\text {in }}=\int_{0}^{l} \psi_{i}(x) \vartheta_{n}(x) d x$. Substituting Fourier series (34) into (32) and (33), we obtain

$$
\left\{\begin{array}{l}
\psi_{1 n}=\int_{0}^{b} \Theta_{1}(t) t^{1-\gamma} \times\left[\varphi_{n} \eta_{1 n}(t, \nu, \omega)+g_{1 n} \eta_{2 n}(t, \nu, \omega)+g_{2 n} \eta_{3 n}(t, \nu, \omega)\right] d t \\
\psi_{2 n}=\int_{-a}^{0} \Theta_{2}(t)\left[\varphi_{n} \xi_{1 n}(t, \nu, \omega)+g_{1 n} \xi_{2 n}(t, \nu, \omega)+g_{2 n} \xi_{3 n}(t, \nu, \omega)\right] d t
\end{array}\right.
$$

This system of equations we rewrite as

$$
g_{1 n} \chi_{i 2 n}(\nu, \omega)+g_{2 n} \chi_{i 3 n}(\nu, \omega)=\psi_{i n}-\varphi_{n} \chi_{i 1 n}(\nu, \omega), \quad i=1,2,
$$

where

$$
\begin{gathered}
\chi_{1 j n}(\nu, \omega)=\int_{0}^{b} \Theta_{1}(t) t^{1-\gamma} \eta_{j n}(t, \nu, \omega) d t, \quad j=1,2,3, \\
\chi_{2 j n}(\nu, \omega)=\int_{-a}^{0} \Theta_{2}(t) \xi_{j n}(t, \nu, \omega) d t, \quad j=1,2,3 .
\end{gathered}
$$

From the system (35) we determine the Fourier coefficients of redefinition functions

$$
\begin{aligned}
& g_{1 n}(\nu, \omega)=\frac{1}{\Delta_{1 n}(\nu, \omega)}\left[\varphi_{n} \tau_{1 n}(\nu, \omega)+\psi_{1 n} \chi_{23 n}(\nu, \omega)-\psi_{2 n} \chi_{13 n}(\nu, \omega)\right] \\
& g_{2 n}(\nu, \omega)=\frac{1}{\Delta_{1 n}(\nu, \omega)}\left[\varphi_{n} \tau_{2 n}(\nu, \omega)-\psi_{1 n} \chi_{22 n}(\nu, \omega)+\psi_{2 n} \chi_{12 n}(\nu, \omega)\right],
\end{aligned}
$$

if there fulfilled condition

$$
\Delta_{1 n}(\nu, \omega)=\chi_{12 n}(\nu, \omega) \chi_{23 n}(\nu, \omega)-\chi_{13 n}(\nu, \omega) \chi_{22 n}(\nu, \omega) \neq 0,
$$

where

$$
\begin{aligned}
& \tau_{1 n}(\nu, \omega)=\chi_{21 n}(\nu, \omega) \chi_{13 n}(\nu, \omega)-\chi_{11 n}(\nu, \omega) \chi_{23 n}(\nu, \omega), \\
& \tau_{2 n}(\nu, \omega)=\chi_{11 n}(\nu, \omega) \chi_{22 n}(\nu, \omega)-\chi_{21 n}(\nu, \omega) \chi_{12 n}(\nu, \omega) .
\end{aligned}
$$

Substituting presentations (36) and (37) into Fourier series (12), we found the redefinition functions

$$
\begin{aligned}
& g_{1}(x, \nu, \omega)=\sum_{n=1}^{\infty} \frac{\vartheta_{n}(x)}{\Delta_{1 n}(\nu, \omega)}\left[\varphi_{n} \tau_{1 n}(\nu, \omega)+\psi_{1 n} \chi_{23 n}(\nu, \omega)-\psi_{2 n} \chi_{13 n}(\nu, \omega)\right] \\
& g_{2}(x, \nu, \omega)=\sum_{n=1}^{\infty} \frac{\vartheta_{n}(x)}{\Delta_{1 n}(\nu, \omega)}\left[\varphi_{n} \tau_{2 n}(\nu, \omega)-\psi_{1 n} \chi_{22 n}(\nu, \omega)+\psi_{2 n} \chi_{12 n}(\nu, \omega)\right]
\end{aligned}
$$

\section{CONVERGENCE OF REDEFINITION FUNCTIONS}

Condition A. Let be

$$
\begin{gathered}
\varphi(x) \in C^{3}[0 ; l], \quad \varphi^{(I V)}(x) \in L(0 ; l), \quad \varphi(0)=\varphi(l)=0 \\
\psi_{i}(x) \in C^{3}[0 ; l], \quad \psi_{i}^{(I V)}(x) \in L(0 ; l), \quad i=1,2 .
\end{gathered}
$$


Then, by integrating in parts desired number of times over the variable $x$ of the integrals

$$
\varphi_{n}=\int_{0}^{l} \varphi(x) \vartheta_{n}(x) d x, \psi_{i n}=\int_{0}^{l} \psi_{i}(x) \vartheta_{n}(x) d x, \quad i=1,2,
$$

we obtain

$$
\begin{aligned}
& \varphi_{n}=\frac{1}{\mu_{n}^{4}} \varphi_{n}^{(I V)}=\frac{1}{\mu_{n}^{4}} \int_{0}^{l} \varphi^{(I V)}(x) \vartheta_{n}(x) d x, \quad \mu_{n}=\frac{n \pi}{l} \\
& \psi_{\text {in }}=\frac{1}{\mu_{n}^{4}} \psi_{i n}^{(I V)}=\frac{1}{\mu_{n}^{4}} \int_{0}^{l} \psi_{i}^{(I V)}(x) \vartheta_{n}(x) d x, \quad i=1,2 .
\end{aligned}
$$

Bessel inequalities are true here

$$
\begin{gathered}
\sum_{n=1}^{\infty}\left[\varphi_{n}^{(I V)}\right]^{2} \leq \frac{2}{l} \int_{0}^{l}\left[\varphi^{(I V)}(x)\right]^{2} d x, \\
\sum_{n=1}^{\infty}\left[\psi_{i n}^{(I V)}\right]^{2} \leq \frac{2}{l} \int_{0}^{l}\left[\psi_{i}^{(I V)}(x)\right]^{2} d x, \quad i=1,2 .
\end{gathered}
$$

Theorem 2. Let conditions $\boldsymbol{A}$ and (38) are fulfilled. Then for regular values of the spectral parameter $\omega$ the Fourier series (39) and (40) are convergent absolutely and uniformly.

Proof. We prove the absolutely and uniformly convergence of series (39). The absolutely and uniformly convergence of series (40) is proved similarly. Taking Lemma 2 into account by the aid of (41)-(44) we obtain

$$
\begin{aligned}
& \left|g_{1}(x, \nu, \omega)\right| \leq M_{2} \sqrt{\frac{l}{2}}\left[\sum_{n=1}^{\infty}\left|\psi_{1 n}\right|+\sum_{n=1}^{\infty}\left|\psi_{2 n}\right|+\sum_{n=1}^{\infty}\left|\varphi_{n}\right|\right] \\
& \leq M_{2} \sqrt{\frac{l}{2}}\left[\sum_{n=1}^{\infty}\left|\frac{1}{\mu_{n}^{4}} \psi_{1 n}^{(I V)}\right|+\sum_{n=1}^{\infty}\left|\frac{1}{\mu_{n}^{4}} \psi_{2 n}^{(I V)}\right|+\sum_{n=1}^{\infty}\left|\frac{1}{\mu_{n}^{4}} \varphi_{n}^{(I V)}\right|\right] \\
& \leq \frac{M_{2} l}{2} \sqrt{\sum_{n=1}^{\infty}\left|\frac{1}{\mu_{n}^{8}}\right|}\left[\sqrt{\sum_{n=1}^{\infty}\left[\psi_{1 n}^{(I V)}\right]^{2}}+\sqrt{\sum_{n=1}^{\infty}\left[\psi_{2 n}^{(I V)}\right]^{2}}+\sqrt{\sum_{n=1}^{\infty}\left[\varphi_{n}^{(I V)}\right]^{2}}\right] \\
& \leq \frac{M_{2} l}{2} \sqrt{\sum_{n=1}^{\infty}\left|\frac{1}{\mu_{n}^{8}}\right|}\left[\sqrt{\int_{0}^{l}\left[\psi_{1}^{(I V)}(x)\right]^{2} d x}\right. \\
& \left.+\sqrt{\int_{0}^{l}\left[\psi_{2}^{(I V)}(x)\right]^{2} d x}+\sqrt{\int_{0}^{l}\left[\varphi^{(I V)}(x)\right]^{2} d x}\right]<\infty
\end{aligned}
$$

where

$$
M_{2}=\max _{n}\left\{\left|\frac{\chi_{23 n}(\nu, \omega)}{\Delta_{1 n}(\nu, \omega)}\right| ;\left|\frac{\chi_{13 n}(\nu, \omega)}{\Delta_{1 n}(\nu, \omega)}\right| ;\left|\frac{\tau_{1 n}(\nu, \omega)}{\Delta_{1 n}(\nu, \omega)}\right|\right\} .
$$

Theorem 2 is proved. 


\section{EXISTENCE OF THE FUNCTION $U(t, x, \nu)$}

Substituting representations (36) and (37) into the Fourier series (25) and (26), respectively, we obtain

$$
\begin{aligned}
& U(t, x, \nu, \omega)=\sum_{n=1}^{\infty} \vartheta_{n}(x)\left[\varphi_{n} \theta_{11 n}(t, \nu, \omega)+\psi_{1 n} \theta_{12 n}(t, \nu, \omega)+\psi_{2 n} \theta_{13 n}(t, \nu, \omega)\right], \quad(t, x) \in \Omega_{1}, \\
& U(t, x, \nu, \omega)=\sum_{n=1}^{\infty} \vartheta_{n}(x)\left[\varphi_{n} \theta_{21 n}(t, \nu, \omega)+\psi_{1 n} \theta_{22 n}(t, \nu, \omega)+\psi_{2 n} \theta_{23 n}(t, \nu, \omega)\right], \quad(t, x) \in \Omega_{2},
\end{aligned}
$$

where

$$
\begin{gathered}
\theta_{11 n}(t, \nu, \omega)=\eta_{1 n}(t, \nu, \omega)+\frac{\eta_{2 n}(t, \nu, \omega)}{\Delta_{1 n}(\nu, \omega)} \tau_{1 n}(\nu, \omega)+\frac{\eta_{3 n}(t, \nu, \omega)}{\Delta_{1 n}(\nu, \omega)} \tau_{2 n}(\nu, \omega), \\
\theta_{12 n}(t, \nu, \omega)=\frac{\eta_{2 n}(t, \nu, \omega)}{\Delta_{1 n}(\nu, \omega)} \chi_{23 n}(\nu, \omega)-\frac{\eta_{3 n}(t, \nu, \omega)}{\Delta_{1 n}(\nu, \omega)} \chi_{22 n}(\nu, \omega) \\
\theta_{13 n}(t, \nu, \omega)=-\frac{\eta_{2 n}(t, \nu, \omega)}{\Delta_{1 n}(\nu, \omega)} \chi_{13 n}(\nu, \omega)+\frac{\eta_{3 n}(t, \nu, \omega)}{\Delta_{1 n}(\nu, \omega)} \chi_{12 n}(\nu, \omega) \\
\theta_{21 n}(t, \nu, \omega)=\xi_{1 n}(t, \nu, \omega)+\frac{\xi_{2 n}(t, \nu, \omega)}{\Delta_{1 n}(\nu, \omega)} \tau_{1 n}(\nu, \omega)+\frac{\xi_{3 n}(t, \nu, \omega)}{\Delta_{1 n}(\nu, \omega)} \tau_{2 n}(\nu, \omega), \\
\theta_{22 n}(t, \nu, \omega)=\frac{\xi_{2 n}(t, \nu, \omega)}{\Delta_{1 n}(\nu, \omega)} \chi_{23 n}(\nu, \omega)-\frac{\xi_{3 n}(t, \nu, \omega)}{\Delta_{1 n}(\nu, \omega)} \chi_{22 n}(\nu, \omega) \\
\theta_{23 n}(t, \nu, \omega)=-\frac{\xi_{2 n}(t, \nu, \omega)}{\Delta_{1 n}(\nu, \omega)} \chi_{13 n}(\nu, \omega)+\frac{\xi_{3 n}(t, \nu, \omega)}{\Delta_{1 n}(\nu, \omega)} \chi_{12 n}(\nu, \omega)
\end{gathered}
$$

Theorem 3. Let conditions $\boldsymbol{A}$ and (38) are fulfilled. Then for regular values of spectral parameter $\omega$ the Fourier series (45) and (46) are convergent absolutely and uniformly. The series (45) and (46) possess the properties (2).

Proof. It is easy to check that for regular values of the spectral parameter $\omega$ there hold for $t \in[0 ; b]$

$$
\begin{gathered}
t^{1-\gamma}\left|u_{n}(t, \nu, \omega)\right| \leq C_{1}\left(\left|\varphi_{n}\right|+\left|\psi_{1 n}\right|+\left|\psi_{2 n}\right|\right), \\
t^{1-\gamma}\left|D^{\alpha, \gamma} u_{n}(t, \nu, \omega)\right| \leq C_{2}\left(\left|\varphi_{n}\right|+\left|\psi_{1 n}\right|+\left|\psi_{2 n}\right|\right)
\end{gathered}
$$

and for $t \in[-a ; 0]$

$$
\begin{gathered}
\left|u_{n}(t, \nu, \omega)\right| \leq C_{3}\left(\left|\varphi_{n}\right|+\left|\psi_{1 n}\right|+\left|\psi_{2 n}\right|\right), \\
\left|\frac{d u_{n}(t, \nu, \omega)}{d t}\right| \leq C_{4}\left(\left|\varphi_{n}\right|+\left|\psi_{1 n}\right|+\left|\psi_{2 n}\right|\right), \\
\left|\frac{d^{2} u_{n}(t, \nu, \omega)}{d t^{2}}\right| \leq C_{5}\left(\left|\varphi_{n}\right|+\left|\psi_{1 n}\right|+\left|\psi_{2 n}\right|\right),
\end{gathered}
$$

where $C_{k}, k=\overline{1,5}$ are positive constants.

Now formally differentiating term-by-term the series (45) and (46) the required number of times, we obtain the series

$$
t^{1-\gamma} D^{\alpha, \gamma} U(t, x, \nu, \omega)=\sum_{n=1}^{\infty} t^{1-\gamma} D^{\alpha, \gamma} u_{n}(t, \nu, \omega) \vartheta_{n}(x), \quad(t, x) \in \Omega_{1},
$$




$$
\begin{gathered}
t^{1-\gamma} \frac{\partial^{k} U(t, x, \nu, \omega)}{\partial x^{k}}=(-1)^{k+1} \sum_{n=1}^{\infty} t^{1-\gamma} u_{n}(t, \nu, \omega) \mu_{n}^{k} \vartheta_{n}(x), \quad k=1,2, \quad(t, x) \in \Omega_{1}, \\
\frac{\partial^{2} U(t, x, \nu, \omega)}{\partial t^{2}}=\sum_{n=1}^{\infty} \frac{d^{2} u_{n}(t, \nu, \omega)}{d t^{2}} \vartheta_{n}(x), \quad(t, x) \in \Omega_{2}, \\
\frac{\partial^{k} U(t, x, \nu, \omega)}{\partial x^{k}}=(-1)^{k+1} \sum_{n=1}^{\infty} u_{n}(t, \nu, \omega) \mu_{n}^{k} \vartheta_{n}(x), \quad k=1,2, \quad(t, x) \in \Omega_{2} .
\end{gathered}
$$

As the case of proof of Theorem 2, it is easy to check the convergence of series (45), (46) and (51)-(54), using the properties (41)-(44) and (47)-(50).

Therefore, for regular values of the spectral parameter $\omega$ the function $U(t, x, \nu, \omega)$, represented by series (45) and (46), possesses properties (2) and satisfies conditions (3)-(7). This is finished the proof of the theorem 3 .

\section{STABILITY OF THE SOLUTION}

For regular values of the spectral parameter $\omega$ we consider the question of the stability of the solution of the inverse boundary value problem $I T_{\nu, \omega}$ with respect to the redefinition functions $g_{i}(x), i=1,2$ and with respect to parameter $\nu$. To this end, we introduce the norm in the space of continuous functions as follows

$$
\|U(t, x, \nu, \omega)\|_{C(\bar{\Omega})}=\max _{(t, x) \in \bar{\Omega}_{1}}\left|t^{1-\gamma} U(t, x, \nu, \omega)\right|+\max _{(t, x) \in \bar{\Omega}_{2}}|U(t, x, \nu, \omega)| .
$$

\subsection{Stability with Respect to Redefinition Functions}

Theorem 4. Suppose that all the conditions of theorem 2 are fulfilled. Then, the solution of the problem $I T_{\nu, \omega}$ for regular values of the spectral parameter $\omega$ is stable with respect to redefinition functions $g_{i}(x), i=1,2$.

Proof. We show that the solution of the mixed differential equation (1) $U(t, x, \nu, \omega)$ is stable with respect to redefinition functions $g_{i}(x), i=1,2$. Let $U_{1}(t, x, \nu, \omega)$ and $U_{2}(t, x, \nu, \omega)$ be two different solutions of the inverse boundary value problem $I T_{\nu, \omega}$, corresponding to two different values of the functions $g_{11}(x), g_{12}(x)$ and $g_{21}(x), g_{22}(x)$.

We put that $\left|g_{11 n}-g_{12 n}\right|<\delta_{1 n},\left|g_{21 n}-g_{22 n}\right|<\delta_{2 n}$, where $0<\delta_{\text {in }}$ are sufficiently small quantities

and the series $\sum_{n=1}^{\infty}\left|\delta_{i n}\right|$ are convergent, $i=1,2$. Then, taking this fact into account, by virtue of the conditions of the theorem, from (25) and (26) we see that

$$
\begin{gathered}
\max _{(t, x) \in \bar{\Omega}_{1}}\left|t^{1-\gamma} U(t, x, \nu, \omega)\right| \leq C_{6} \sum_{n=1}^{\infty}\left(\left|g_{11 n}-g_{12 n}\right|+\left|g_{21 n}-g_{22 n}\right|\right) \\
<C_{6} \sum_{n=1}^{\infty}\left(\left|\delta_{1 n}\right|+\left|\delta_{2 n}\right|\right) \\
\max _{(t, x) \in \bar{\Omega}_{2}}|U(t, x, \nu, \omega)| \leq C_{7} \sum_{n=1}^{\infty}\left(\left|g_{11 n}-g_{12 n}\right|+\left|g_{21 n}-g_{22 n}\right|\right) \\
<C_{7} \sum_{n=1}^{\infty}\left(\left|\delta_{1 n}\right|+\left|\delta_{2 n}\right|\right) \\
|| U_{1}(t, x, \nu, \omega)-U_{2}(t, x, \nu, \omega)||_{C(\bar{\Omega})}<C_{8} \sum_{n=1}^{\infty}\left(\left|\delta_{1 n}\right|+\left|\delta_{2 n}\right|\right)
\end{gathered}
$$


where $C_{8}=C_{6}+C_{7}, C_{6}=\max \left\{C_{6.1} ; C_{6.2} ; C_{6.3}\right\}, C_{7}=\max \left\{C_{7.1} ; C_{7.2} ; C_{7.3}\right\}$,

$$
C_{6 . i}=\max _{n \in \mathbb{N}} \max _{0<t<b}\left|t^{1-\gamma} \eta_{i n}(t, \nu, \omega)\right| ; \quad C_{7 . i}=\max _{n \in \mathbb{N}} \max _{-a<t<0}\left|\xi_{i n}(t, \nu, \omega)\right|, \quad i=1,2,3 .
$$

By virtue of these estimates we finally obtain assertions on the stability of the solution of differential equation (1) with respect to redefinition functions $g_{i}(x), i=1,2$, if we put $\varepsilon=C_{8} \sum_{n=1}^{\infty}\left(\left|\delta_{1 n}\right|+\left|\delta_{2 n}\right|\right)$. The Theorem 4 is proved.

\subsection{Stability with Respect To Parameter $\nu$}

Now we show that the solution of the mixed differential equation (1) $U(t, x, \nu, \omega)$ is stable with respect to a given parameter $\nu$.

Theorem 5. Suppose that all the conditions of theorem 2 are fulfilled. Then, the solution of the problem $I T_{\nu, \omega}$ for regular values of the spectral parameter $\omega$ is stable with respect to given parameter $\nu$.

Proof. Let $U_{1}(t, x, \nu, \omega)$ and $U_{2}(t, x, \nu, \omega)$ be two different solutions of the inverse boundary value problem $I T_{\nu, \omega}$, corresponding to two different values of the parameter $\nu_{1}$ and $\nu_{1}$, respectively. We put that $\left|\nu_{1}-\nu_{2}\right|<\delta$, where $0<\delta$ is sufficiently small real number. By virtue of $\lambda_{n}^{2}(\nu)=\frac{\mu_{n}^{2}}{1+\nu \mu_{n}^{2}}$, we derive the following estimates

$$
\begin{aligned}
& \| h_{1 n}\left(t, \nu_{1}\right)-h_{1 n}\left(t, \nu_{2}\right)|| \leq \max _{t} \int_{0}^{t}\left|(t-s)^{\alpha-1} f_{1}(s)\right| \cdot \mid E_{\alpha, \alpha}\left(-\lambda_{n}^{2}\left(\nu_{1}\right)(t-s)^{\alpha}\right) \\
& -E_{\alpha, \alpha}\left(-\lambda_{n}^{2}\left(\nu_{2}\right)(t-s)^{\alpha}\right)\left|d s \leq C_{9}\right| \lambda_{n}^{2}\left(\nu_{1}\right)-\lambda_{n}^{2}\left(\nu_{2}\right) \mid \\
& \leq C_{9}\left|\int_{\nu_{1}}^{\nu_{2}} \frac{d}{d \nu} \frac{\mu_{n}^{2}}{1+\nu \mu_{n}^{2}}\right| \leq C_{10}\left|\nu_{1}-\nu_{2}\right| \\
& \left\|h_{2 n}\left(t, \nu_{1}\right)-h_{2 n}\left(t, \nu_{2}\right)\right\| \\
& \leq \frac{1}{\omega} \max _{t} \int_{0}^{t}\left|f_{2}(s)\right|\left|\frac{\sin \lambda_{n}\left(\nu_{1}\right) \omega(t-s)}{\lambda_{n}\left(\nu_{1}\right)}-\frac{\sin \lambda_{n}\left(\nu_{2}\right) \omega(t-s)}{\lambda_{n}\left(\nu_{2}\right)}\right| d s \\
& \leq \frac{1}{\omega} \max _{t} \int_{0}^{t}\left|f_{2}(s)\right|\left[\left|\int_{\nu_{1}}^{\nu_{2}} \frac{d}{d \nu} \frac{1}{\lambda_{n}(\nu)}\right|\right. \\
& \left.+C_{11}\left|\int_{\nu_{1}}^{\nu_{2}} \frac{d}{d \nu} \sin \lambda_{n}(\nu) \omega(t-s)\right|\right] d s \leq C_{12}\left|\nu_{1}-\nu_{2}\right| \\
& \| \lambda_{n}\left(\nu_{1}\right) \sin \lambda_{n}\left(\nu_{1}\right) \omega t-\lambda_{n}\left(\nu_{2}\right) \sin \lambda_{n}\left(\nu_{2}\right) \omega t|| \leq \max _{t}\left|\left[\lambda_{n}\left(\nu_{1}\right)-\lambda_{n}\left(\nu_{2}\right)\right] \sin \lambda_{n}\left(\nu_{2}\right) \omega t\right| \\
& +\left|\lambda_{n}\left(\nu_{1}\right) \max _{t}\left[\sin \lambda_{n}\left(\nu_{1}\right) \omega t-\sin \lambda_{n}\left(\nu_{2}\right) \omega t\right]\right| \leq C_{13}\left|\nu_{1}-\nu_{2}\right|,
\end{aligned}
$$

where $C_{9}, C_{10}, \ldots, C_{13}$ are constants. Similarly, we can show that the following estimate is also valid

$$
\left\|\theta_{i j n}\left(t, \nu_{1}, \omega\right)-\theta_{i j n}\left(t, \nu_{2}, \omega\right)\right\| \leq C_{14}\left|\nu_{1}-\nu_{2}\right|, \quad C_{14}=\text { const }, \quad i=1,2, \quad j=1,2,3 .
$$

Therefore, for series (45) and (46) we obtain

$$
\begin{gathered}
\left\|U_{1}(t, x, \nu, \omega)-U_{2}(t, x, \nu, \omega)\right\|_{C(\bar{\Omega})} \\
\leq C_{15}\left[\left\|\varphi^{(I V)}(x)\right\|_{C[0 ; l]}+\left\|\psi_{1}^{(I V)}(x)\right\|_{C[0 ; l]}+\left\|\psi_{2}^{(I V)}(x)\right\|_{C[0 ; l]}\right]\left|\nu_{1}-\nu_{2}\right|
\end{gathered}
$$




$$
<C_{15}\left[\left\|\varphi^{(I V)}(x)\right\|_{C[0 ; l]}+\left\|\psi_{1}^{(I V)}(x)\right\|_{C[0 ; l]}+\left\|\psi_{2}^{(I V)}(x)\right\|_{C[0 ; l]}\right] \delta,
$$

where $C_{15}$ is constant. If we put

$$
\varepsilon=C_{15}\left[\left\|\varphi^{(I V)}(x)\right\|_{C[0 ; l]}+\left\|\psi_{1}^{(I V)}(x)\right\|_{C[0 ; l]}+\left\|\psi_{2}^{(I V)}(x)\right\|_{C[0 ; l]}\right] \delta,
$$

then we obtain

$$
\left\|U_{1}(t, x, \nu, \omega)-U_{2}(t, x, \nu, \omega)\right\|_{C(\bar{\Omega})}<\varepsilon
$$

The Theorem 5 is proved.

\section{IRREGULAR VALUES OF SPECTRAL PARAMETER $\omega$}

We note that $\Delta_{m}(\omega, \nu)=0$ for irregular values of the spectral parameter $\omega$ and $m=k_{1}, \ldots, k_{s}$ $(\gamma \neq 1)$. Then, for the solvability of systems (18) and (19), it is necessary and sufficient that the orthogonality conditions are satisfied

$$
\varphi_{m}=\int_{0}^{l} \varphi(x) \vartheta_{m}(x) d x=0 .
$$

In this case, by virtue of (27), the solutions of direct problem are represented as the sum of the series

$$
\begin{gathered}
U(t, x)=\sum_{m=1}^{\infty} C_{m} \vartheta_{m}(x)\left[t^{\gamma-1} E_{\alpha, \gamma}\left(-\lambda_{m}^{2}(\nu) t^{\alpha}\right)+g_{1 m} h_{1 m}(t, \nu)\right], \quad(t, x) \in \Omega_{1}, \\
U(t, x)=\sum_{m=1}^{\infty} C_{m} \vartheta_{m}(x)\left[\sin \lambda_{m}(\nu) \omega t+\cos \lambda_{m}(\nu) \omega t+g_{2 m} h_{2 m}(t, \nu)\right], \quad(t, x) \in \Omega_{2},
\end{gathered}
$$

where $m=k_{1}, \ldots, k_{s}, C_{m}$ are arbitrary constants.

For irregular values of the spectral parameter $\omega$ and for some $m=k_{1}, \ldots, k_{s}$ direct problem has an infinite number of solutions in the form of series (56) and (57). The solvability condition is formula (55). Substituting Fourier series (56) and (57) into (6) and (7), respectively, we obtain

$$
\begin{gathered}
\psi_{1}(x)=\sum_{m=1}^{\infty} C_{m} \vartheta_{m}(x) \\
\times \int_{0}^{b} \Theta_{1}(t) t^{1-\gamma}\left[t^{\gamma-1} E_{\alpha, \gamma}\left(-\lambda_{m}^{2}(\nu) t^{\alpha}\right)+g_{1 m} h_{1 m}(t, \nu)\right], \quad(t, x) \in \Omega_{1}, \\
\psi_{2}(x)=\sum_{m=1}^{\infty} C_{m} \vartheta_{m}(x) \\
\times \int_{-a}^{0} \Theta_{2}(t)\left[\sin \lambda_{m}(\nu) \omega t+\cos \lambda_{m}(\nu) \omega t+g_{2 m} h_{2 m}(t, \nu)\right], \quad(t, x) \in \Omega_{2},
\end{gathered}
$$

where $m=k_{1}, \ldots, k_{s}$.

By virtue of (34), from (58) and (59) we derive coefficients of redefinition functions

$$
g_{1 m}=\sigma_{1 m}=\frac{\psi_{1 m}-C_{m} \int_{0}^{b} \Theta_{1}(t) E_{\alpha, \gamma}\left(-\lambda_{m}^{2}(\nu) t^{\alpha}\right) d t}{C_{m} \int_{0}^{b} \Theta_{1}(t) t^{1-\gamma} h_{1 m}(t, \nu) d t},
$$




$$
g_{2 m}=\sigma_{2 m}=\frac{\psi_{2 m}-C_{m} \int_{-a}^{0} \Theta_{2}(t)\left(\sin \lambda_{m}(\nu) \omega t+\cos \lambda_{m}(\nu) \omega t\right) d t}{C_{m} \int_{-a}^{0} \Theta_{2}(t) h_{2 m}(t, \nu) d t},
$$

where

$$
\int_{0}^{b} \Theta_{1}(t) t^{1-\gamma} h_{1 m}(t, \nu) d t \neq 0, \quad \int_{-a}^{0} \Theta_{2}(t) h_{2 m}(t, \nu) d t \neq 0 .
$$

Substituting Fourier coefficients (60) and (61) into series (12), (56) and (57) we obtain Fourier series for redefinition functions $g_{1}(x), g_{2}(x)$ and for main unknown function $U(t, x)$ :

$$
\begin{gathered}
g_{i}(x)=\sum_{m=1}^{\infty} \sigma_{i m} \vartheta_{m}(x), \quad i=1,2 ; \\
U(t, x)=\sum_{m=1}^{\infty} C_{m} \vartheta_{m}(x) \\
\times\left[t^{\gamma-1} E_{\alpha, \gamma}\left(-\lambda_{m}^{2}(\nu) t^{\alpha}\right)+\sigma_{1 m} h_{1 m}(t, \nu)\right], \quad(t, x) \in \Omega_{1}, \\
U(t, x)=\sum_{m=1}^{\infty} C_{m} \vartheta_{m}(x) \\
\times\left[\sin \lambda_{m}(\nu) \omega t+\cos \lambda_{m}(\nu) \omega t+\sigma_{2 n} h_{2 n}(t, \nu)\right], \quad(t, x) \in \Omega_{2},
\end{gathered}
$$

where $m=k_{1}, \ldots, k_{s}$ and $C_{m}$ are arbitrary constants.

The absolutely and uniformly convergence of obtained series (63)-(65) is clear. Because $C_{m}$ are arbitrary numbers. Them we can select so that these series (63)-(65) converge. The coefficients $\psi_{1 m}$ and $\psi_{2 m}$ in (60) and (61) are satisfy the properties (42) and (44).

\section{CONCLUSIONS}

We studied the inverse boundary value problem $I T_{\nu, \omega}$ with following assumption

$$
\begin{gathered}
\varphi(x) \in C^{3}[0 ; l], \varphi^{(I V)}(x) \in L(0 ; l), \quad \varphi(0)=\varphi(l)=0, \\
\psi_{i}(x) \in C^{3}[0 ; l], \quad \psi_{i}^{(I V)}(x) \in L(0 ; l), \quad i=1,2 .
\end{gathered}
$$

If these conditions and (38) are fulfilled, then the inverse boundary value problem $I T_{\nu, \omega}$ is uniquely solvable for regular values of the spectral parameter $\omega \in \aleph$ and this solution is represented in the form of the Fourier series (39), (40) and (45), (46) in the domain $\Omega$.

For irregular values of the spectral parameter $\omega \in \Im$ and for some $m=k_{1}, \ldots, k_{s}$ inverse problem $I T_{\nu, \omega}$ has an infinite number of solutions in the form of series (63)-(65), if there the condition (62) is fulfilled. The solvability condition is formula (55).

For regular values of the spectral parameter $\omega$ we studied also the questions of the stability of the solution of the inverse boundary value problem $I T_{\nu, \omega}$ with respect to the redefinition functions $g_{1}(x)$, $g_{2}(x)$ and with respect to parameter $\nu$. 


\section{REFERENCES}

1. C. Xu, Y. Yu, Y. Q. Chen, and Z. Lu, "Forecast analysis of the epidemic trend of COVID-19 in the United States by a generalized fractional-order SEIR model," arXiv: 2004.12541v1 (2020).

2. Handbook of Fractional Calculus with Applications, Ed. by J. A. Tenreiro Machado (Walter de Gruyter, Berlin, Boston, 2019).

3. D. Kumar and D. Baleanu, "Fractional calculus and its applications in physics," Front. Phys. 7 (6) (2019).

4. M.-H. Kim, G.-Ch. Ri, and O. Hyong-Chol, "Operational method for solving multi-term fractional differential equations with the generalized fractional derivatives," Fract. Calc. Appl. Anal. 17, 79-95 (2014).

5. S. Patnaik, J. P. Hollkamp, and F. Semperlotti, "Applications of variable-order fractional operators: A review," Proc. R. Soc. London, Ser. A 476, 20190498 (2020).

6. R. K. Saxena, R. Garra, and E. Orsingher, "Analytical solution of space-time fractional telegraph-type equations involving Hilfer and Hadamard derivatives," Integr. Transforms Spec. Funct. 6 (2015).

7. H. Sun, A. Chang, Y. Zhang, and W. Chen, "A review on variable-order fractional differential equations: Mathematical foundations, physical models, numerical methods and applications," Fract. Calculus Appl. Anal. 22, 27-59 (2019).

8. S. A. Malik and S. Aziz, "An inverse source problem for a two parameter anomalous diffusion equation with nonlocal boundary conditions," Comput. Math. Appl. 73 (12)(2017).

9. S. Aziz and S. A. Malik, "Identification of an unknown source term for a time fractional fourth-order parabolic equation," Electron. J. Differ. Equat. 2016 (293), 1-20 (2016).

10. K. B. Sabitov and S. N. Sidorov, "On a nonlocal problem for a degenerating parabolic-hyperbolic equation," Differ. Equat. 50, 352-361 (2014).

11. K. B. Sabitov, On the Theory of Mixed Type Equations (Fizmatlit, Moscow, 2014) [in Russian].

12. T. K. Yuldashev, "Solvability of a boundary value problem for a differential equation of the Boussinesq type," Differ. Equat. 54, 1384-1393 (2018).

13. T. K. Yuldashev, "A coefficient determination in nonlocal problem for Boussinesq type integro-differential equation with degenerate kernel," Vladikavk. Math. J. 21 (2), 67-84 (2019).

14. T. K. Yuldashev, "On a boundary-value problem for Boussinesq type nonlinear integro-differential equation with reflecting argument,” Lobachevskii J. Math. 41 (1), 111-123 (2020).

15. T. K. Yuldashev, "Nonlocal inverse problem for a pseudohyperbolic-pseudoelliptic type integro-differential equations," Axioms 9, (2), 45-1-21 (2020).

16. O. S. Zikirov, "A non-local boundary value problem for third-order linear partial differential equation of composite type," Math. Model. Anal. 14, 407-421 (2009).

17. A. S. Berdyshev and B. J. Kadirkulov, "On a nonlocal problem for a fourth-order parabolic equation with the fractional Dzhrbashyan-Nersesyan operator,” Differ. Equat. 52, 122-127 (2016).

18. A. S. Berdyshev, A. Cabada, and B. J. Kadirkulov, "The Samarskii-Ionkin type problem for fourth order parabolic equation with fractional differential operator," Comput. Math. Appl. 62, 3884-3893 (2011).

19. S. Kerbal, B. J. Kadirkulov, and M. Kirane, "Direct and inverse problems for a Samarskii-Ionkin type problem for a two dimensional fractional parabolic equation,” Progr. Fract. Differ. Appl. 4 (3), 1-14 (2018).

20. T. K. Yuldashev and B. J. Kadirkulov, "Boundary value problem for weak nonlinear partial differential equations of mixed type with fractional Hilfer operator," Axioms 9 (2), 68-1-19 (2020). 